\title{
Spectral Analyses of Wolf-Rayet Stars: The Impact of Clumping
}

\author{
Wolf-Rainer Hamann and Lars Koesterke \\ Universität Potsdam, Am Neuen Palais 10, D-14469 Potsdam, Germany
}

\begin{abstract}
Inhomogeneities are accounted for in our non-LTE stellar wind models in a first-order approximation. When applied for spectral analyses, clumpy models yield lower mass-loss rates than homogeneous models, while other parameters are not affected. For representative WR stars, we determine the density contrast from the electron-scattering line wings and obtain mass-loss rate reductions by a factor of two, typically.
\end{abstract}

\section{Model atmospheres with clumping}

The so-called standard models for WR atmospheres are based on the assumptions of spherical symmetry, homogeneity and stationarity of the flow. The main features of WR spectra can be reproduced by these calculations, thus validating its basic assumptions as a reasonable approximation. However, there are various evidences that real WR atmospheres are actually inhomogeneous to some degree.

For a first-order approach to clumped stellar winds, we implement the following simplifying treatment of clumping in our non-LTE model atmosphere code. In the clumps the density is enhanced by the factor $D$ with respect to the homogeneous model with the same mass-loss rate. The interclump space is void. The clumps are assumed to have small size, compared to the photon free path.

In the described formulation, the density enhancement $D$ would cancel out in the emissivities and opacities, if they were linear in density. However, Wolf-Rayet spectra are known to be dominated by processes which scale with the square of the density. This can be concluded from the scaling property of Wolf-Rayet models, which has been discovered first by Schmutz et al. (1989). They defined a so-called transformed radius $R_{\mathrm{t}}$ as a combination of $\dot{M}, R_{*}$ and $v_{\infty}$ and found that models with same $R_{\mathrm{t}}$ exhibit the same emission line equivalent widths, irrespective of different combinations of the involved parameters (while, of course, $T_{*}$, composition etc. are fixed). This invariance was validated by various numerical experiments with reasonable accuracy.

The concept of the "transformed radius" now is generalized for clumped models. In order to cancel out in quadratic processes, the clump density enhancement $D$ must be compensated by diminishing the mass-loss rate by a factor $\sqrt{D}$. Thus we define 


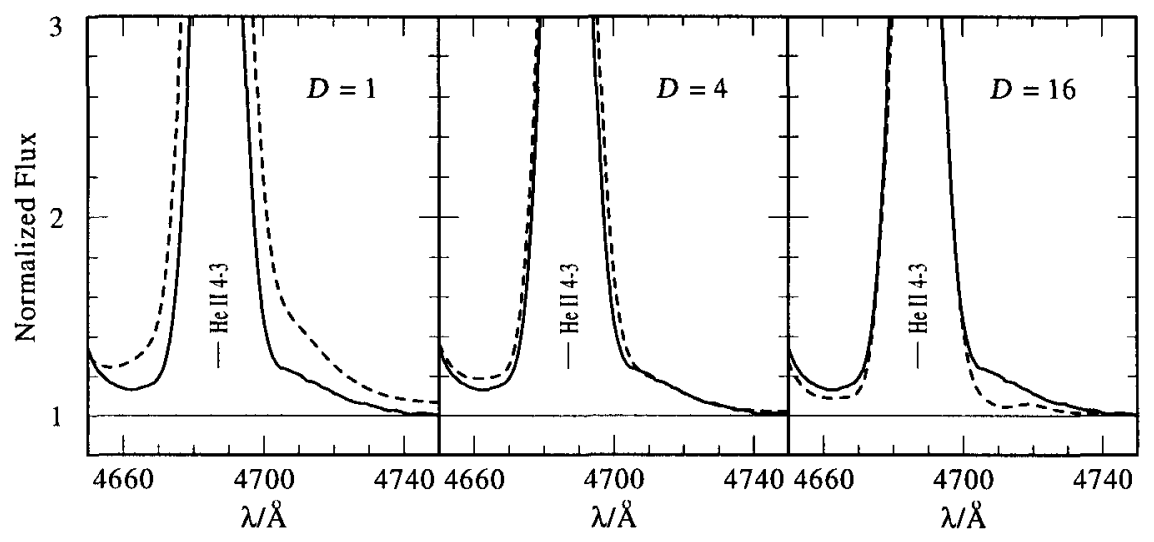

Fig. 1. The line wings of the He II line at $4686 \AA$, as observed in the WN7-type LMC star Br 24 (solid line). The synthetic profiles (dashed lines) were calculated for different density contrast $D$ as indicated. The mass-loss rate is scaled with $D^{-0.5}$ for compensation, while the other parameters (see caption of Fig. 2) are maintained.

$$
R_{\mathrm{t}}=R_{*}\left[\frac{v_{\infty}}{2500 \mathrm{~km} \mathrm{~s}^{-1}} / \frac{\sqrt{D} \dot{M}}{10^{-4} M_{\odot} \mathrm{yr}^{-1}}\right]^{2 / 3}
$$

and expect that models with same $R_{\mathrm{t}}$ exhibit the same line equivalent widths, irrespective of different combinations of $D, \dot{M}, R_{*}$ and $v_{\infty}$. For constant $v_{\infty}$, the absolute spectra should only differ by a scaling with $R_{*}^{2}$.

Note that this scaling invariance holds only for the $\rho^{2}$ processes, i.e. for the main spectral features. The electron scattering opacity, however, scales linearly with density. Thus, in inhomogeneous models the enhanced clump density is already fully compensated by the volume filling factor; scaling down the mass-loss rate in order to keep the same $R_{\mathrm{t}}$ decreases the effective Thomson opacity. Now, Thomson scattering causes a wide frequency redistribution of photons, due to the high thermal speed of the electrons (cf. Hillier 1984). Hence, for a series of models with same $R_{\mathrm{t}}$ the extended line wings caused by this frequency redistribution of electron-scattered line photons become weaker with increasing $D$, while the main spectral feature remain unchanged.

\section{Spectral fits and conclusions}

The described dependence of the electron-scattering line wings can be used to determine the adequate value of $D$ from observed spectra. This is demonstrated here by the example of $\mathrm{Br} 24$. The comparison with the observation (Fig. 1) reveals that the homogeneous model $(D=1)$ gives too strong electron-scattering wings, while the model with $D=4$ fits best. 


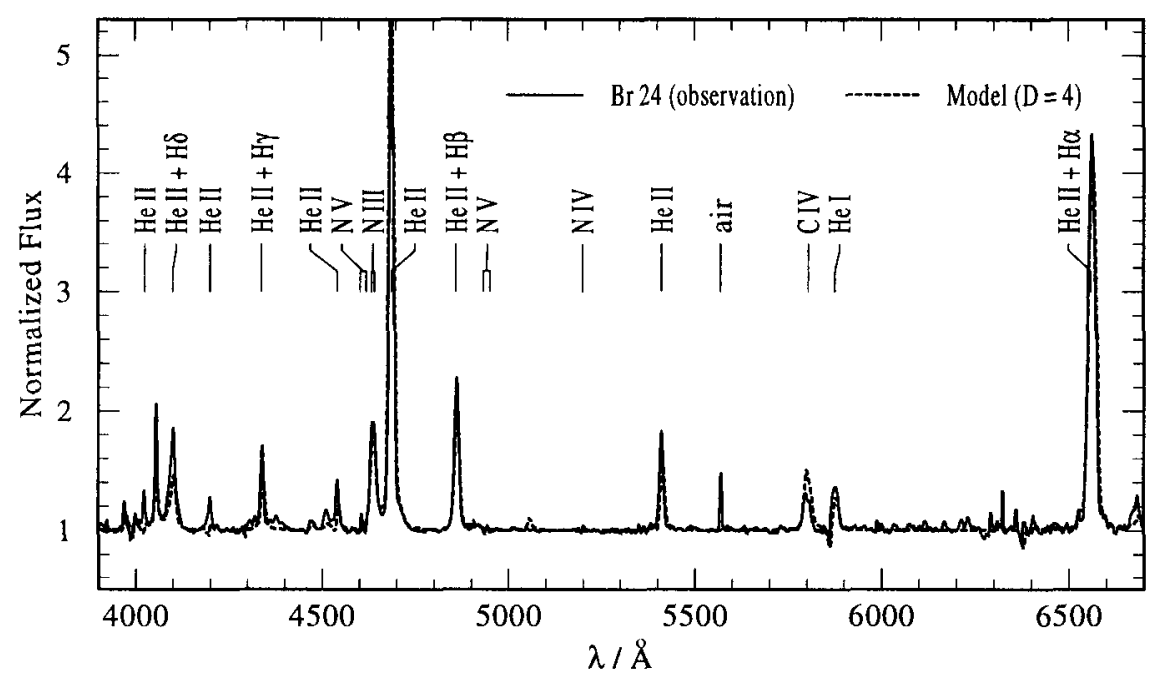

Fig. 2. The observed spectrum of the LMC star Br 24 in the visual, compared to a model calculation with clumping $(D=4)$. Accounting for the clumping effect has nicely improved the detailed agreement between observed WR spectra and our models. Model parameters: $T_{*}=40 \mathrm{kK}, \log L / L_{\odot}=5.35, \log \dot{M} /\left(M_{\odot} \mathrm{yr}^{-1}\right)=-5.0$, $v_{\infty}=950 \mathrm{~km} / \mathrm{s}$, composition $\mathrm{H} / \mathrm{He} / \mathrm{C} / \mathrm{N}=40 / 59 / 0.01 / 0.8$ (by mass)

To the first order, the mass-loss rate is the only parameter which is affected by the application of clumped models for spectral analyses. In a recent paper (Hamann \& Koesterke 1998b) we selected representative Galactic WR stars of different spectral subclass and found that $D=4$ is a typical value, which implies that the empirical mass-loss rates become smaller by factor $\sqrt{D}=2$ than obtained from the use of homogeneous models. In Hamann \& Koesterke (1998a) we analyzed the nitrogen spectra of the Galactic WN stars. If their mass-loss rates are now scaled down by a factor of two, the average values of the "momentum ratio" $\dot{M} v_{\infty} c / L$ become $4.5,4.3$ and 15 for the WNL, WNE-w and WNE-s spectral subclass, respectively. These values are still above the single-scattering limit (i.e. unity), but no longer implausible for radiative acceleration with multiple-scattering effects. The consequences of smaller WR mass-loss rates for the stellar evolution must be considered.

\section{References}

Hamann W.-R., Koesterke L., 1998a, A\&A 333, 251

Hamann W.-R., Koesterke L., 1998b, A\&A 335, 1003

Hillier D.J., 1984, ApJ 280, 744

Schmutz W., Hamann W.-R., Wessolowski U., 1989, A\&A 210, 236 


\section{Discussion}

G. Koenigsberger: Regarding the clumping factor, which appears to be the same for all WR subclasses: could you comment on why "D" should be independent of the properties that determine the wind structure, which in turn lead to the observed spectra?

W.-R. Hamann: Clumping in WR winds is most likely caused by the instability of radiation driving. Existing calculations (Gayley \& Owocki 1995, ApJ 446,801) show that the perturbation growth rate scales with the inverse of the wind momentum ratio, $\eta$. Although $\eta$ is larger for WR stars than for OB stars, the instability is predicted to produce strong clumping. However, fully self-consistent hydrodynamical WR models do not yet exist. Thus, we have no prediction about how the density contrast may depend on the stellar parameters.

R. Ignace: Recent work by Nugis et al. suggests that the free-free continuum slopes of WR stars cannot be explained by constant clumping and filling factors. Can you comment on the consequences of their inferences for your profile modelling?

W.-R. Hamann: Free-free radio emission is a $\rho^{2}$-process, as is the line emission. Hence radio mass loss rates are affected by clumping in exactly the same way as shown in my talk. The mass-loss rates derived from radio emission agree fairly well with those from optical/UV lines. This implies that the clumping contrast must be similar in the radio-emitting region (far out in the stellar wind) and the line-forming region, respectively. The slope of the radio free-free continuum is not affected by clumping, unless the density contrast strongly varies with the radial coordinate. The corresponding evidence given by Nugis et al. (1998, A\&A 333, 956) is rather weak in my opinion.

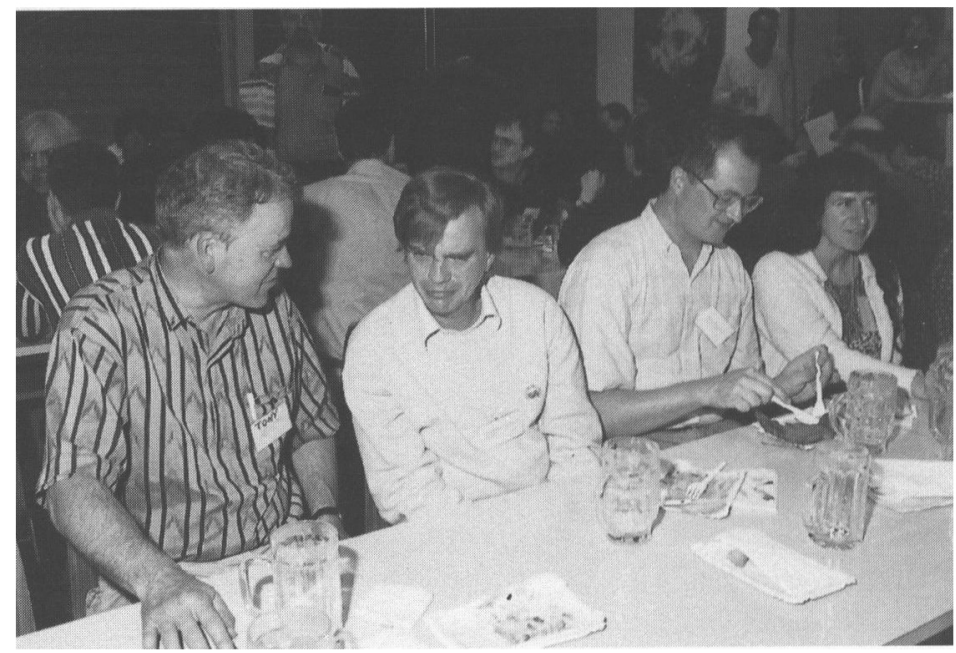

Anthony Moffat, Wolf-Rainer Hamann, Knut Ødegaard and Joyce Guzik 